\title{
Midinfrared photoreflectance study of InAs-rich InAsSb and GalnAsPSb indicating negligible bowing for the spin orbit splitting energy
}

\author{
S. A. Cripps and T. J. C. Hosea ${ }^{a)}$ \\ Department of Physics, University of Surrey, Guildford, Surrey GU2 7XH, United Kingdom
}

A. Krier, V. Smirnov, P. J. Batty, and Q. D. Zhuang

Department of Physics, Lancaster University, Lancaster LAl 4YB, United Kingdom

H. H. Lin, Po-Wei Liu, and G. Tsai

Department of Electronic Engineering, National Taiwan University, Taipei 106, Taiwan

(Received 18 January 2007; accepted 21 March 2007; published online 24 April 2007)

\begin{abstract}
The authors report on midinfrared photoreflectance measurements of the band gap $\left(E_{0}\right)$ and spin-orbit splitting energies $\left(\Delta_{0}\right)$ in InAs-rich InAsSb and GaInAsPSb samples for varying antimony contents $\leqslant 22.5 \%$. The $E_{0}$ behavior as a function of $\mathrm{Sb}$ content is consistent with the literature value bowing parameter of $\sim 670 \mathrm{meV}$. However, $\Delta_{0}$ does not exhibit the positive bowing of $+1170 \mathrm{meV}$ quoted in the literature: rather, a best fit to their data tentatively suggests a negative bowing of $-225 \mathrm{meV}$. This result is likely to have strong impact due to the importance of the $\Delta_{0}$ parameter in governing InAsSb-based device performance. () 2007 American Institute of Physics.
\end{abstract}

[DOI: $10.1063 / 1.2728752$ ]

Recent interest in developing midinfrared (MIR, $2-5 \mu \mathrm{m})$ light emitters and detectors has been driven by the wide range of potential applications in this spectral region. ${ }^{1}$ As a result of high Auger recombination coefficients, MIR semiconductors exhibit low quantum efficiencies and devices often have to operate below room temperature. ${ }^{2}$ The Auger recombination rates are sensitive to the fundamental band gap energy $E_{0}$ and [in the case of the conduction, heavy-hole, spin split-off hole, heavy-hole (CHSH) process] the spinorbit splitting energy $\Delta_{0}{ }^{3}$ Therefore, in order to evaluate losses by these nonradiative recombination paths, a good knowledge of both $E_{0}$ and $\Delta_{0}$ is essential.

The $\operatorname{InAs}_{1-x} \mathrm{Sb}_{x}$ alloy plays a particularly important role in many MIR devices. ${ }^{2,45}$ As a ternary alloy it is possible to define the compositional dependence of a parameter $T$ (such as $E_{0}$ or $\left.\Delta_{0}\right)$ using $T=x B_{1}+(1-x) B_{2}-x(1-x) C$, where $B_{1}$ is the binary end-point InSb value, $B_{2}$ the InAs value, and $C$ a bowing parameter which describes any deviation from the virtual crystal approximation. ${ }^{6}$ A range of studies of $E_{0}$ in $\operatorname{InAs}_{1-x} \mathrm{Sb}_{x}$ indicates that $C$ lies between $570-690 \mathrm{meV}^{7-9}$ and the review of Vurgaftman et al. recommends using $C\left(E_{0}\right)=+670 \mathrm{meV} .{ }^{10}$ There is less information in the literature, and some uncertainty, regarding $\Delta_{0}$ in $\operatorname{InAs}_{1-x} \mathrm{Sb}_{x}$. In 1972 Berolo and Woolley ${ }^{11}$ published the first and only measured values for $\Delta_{0}$, using electroreflectance on samples grown by the horizontal Bridgman technique. ${ }^{12}$ Although no actual spectra were published, they reported a bowing parameter of $C\left(\Delta_{0}\right)=+1170 \mathrm{meV}$, by far the largest of any III-V ternary alloy. ${ }^{10}$ They attributed this, and similar trends they observed in other ternary alloys, ${ }^{11}$ to interband mixing effects and disordering within the lattice. ${ }^{13}$ Subsequently, several authors have suggested that such bowing $\left[C\left(\Delta_{0}\right)\right.$ $>0]$ is not predicted by theory for ordered systems: Siggia remarks that his calculations for $\Delta_{0}$ in $\operatorname{InAs}_{1-x} \mathrm{Sb}_{x}$ cannot demonstrate the bowing claimed by Berolo and Woolley ${ }^{11}$ unless his Kane-formula conduction band effective mass val-

\footnotetext{
${ }^{a)}$ Electronic mail: j.hosea@surrey.ac.uk
}

ues are doubled, but that would then disagree with experimental measurements of the effective mass. ${ }^{14}$ After several experiments reported $C\left(\Delta_{0}\right)<0$ in epitaxial quaternary alloys, ${ }^{15-17}$ Wei and Zunger ${ }^{18}$ conducted self-consistent electronic-structure calculations for ordered ternary alloys which predicted $C\left(\Delta_{0}\right)<0$. They noted that an unphysically large interband mixing coefficient of $48 \%$ would be required to explain the positive bowing in $\operatorname{InAs}{ }_{1-x} \mathrm{Sb}_{x}$ and concluded instead that sample inhomogeneity in Ref. 11 was a more likely cause, with a recommendation to conduct new measurements on homogeneous epitaxial samples. Here, we use MIR photoreflectance (PR) spectroscopy to measure the $E_{0}$ and $E_{0}+\Delta_{0}$ transitions in high-quality epitaxial $\operatorname{InAs}_{1-x} \mathrm{Sb}_{x}$ samples (of thickness $500 \mathrm{~nm}$, with $300 \mathrm{~nm}$ InAs buffer layers) grown by molecular beam epitaxy (MBE), on $n$-InAs substrates, with $\mathrm{Sb}$ molar fractions of $0 \leqslant x \leqslant 22.5 \%$ determined from $\mathrm{x}$-ray diffraction measurements. In all these samples the epilayers are thicker than the determined critical thicknesses $(\sim 100 \mathrm{~nm})$, indicating that they are likely to be fully relaxed. We also study the PR of two InAsSb-rich, $\mathrm{Ga}_{z} \mathrm{In}_{1-z} \mathrm{As}_{1-y-x} \mathrm{P}_{y} \mathrm{Sb}_{x}$ samples of thickness $\sim 1.5 \mu \mathrm{m}$, grown by liquid phase epitaxy (LPE), lattice matched to $\mathrm{GaSb}$ substrates. These two pentenary alloys ( $s 922$ and $s 923$ ) were determined to have the compositions $\mathrm{Ga}_{0.04} \mathrm{In}_{0.96} \mathrm{As}_{0.83} \mathrm{P}_{0.04} \mathrm{Sb}_{0.13}$ and $\mathrm{Ga}_{0.07} \mathrm{In}_{0.93} \mathrm{As}_{0.82} \mathrm{P}_{0.03} \mathrm{Sb}_{0.15}$, respectively.

The PR was done using a $0.32 \mathrm{~m}$ monochromator with gratings blazed at either 1.5 or $5.0 \mu \mathrm{m}$ to disperse the probe beam from a tungsten filament bulb, and detected by a $77 \mathrm{~K}$ $\mathrm{InSb}$ photodiode. The sample reflectivity was modulated with a mechanically chopped $(883 \mathrm{~Hz}) \mathrm{HeNe}$ laser $(633 \mathrm{~nm}$, $\sim 10 \mathrm{~mW}$ ). In some samples strong background signals necessitated a reduction of the laser power with neutral density filters.

Figure 1 shows the PR of all our samples at $10 \mathrm{~K}$, with the exception of the $16.0 \% \mathrm{Sb}$ sample, which exhibited a particularly weak PR signal: here a better signal to noise ratio was achieved at $50 \mathrm{~K}$. This decreases its band gap by 


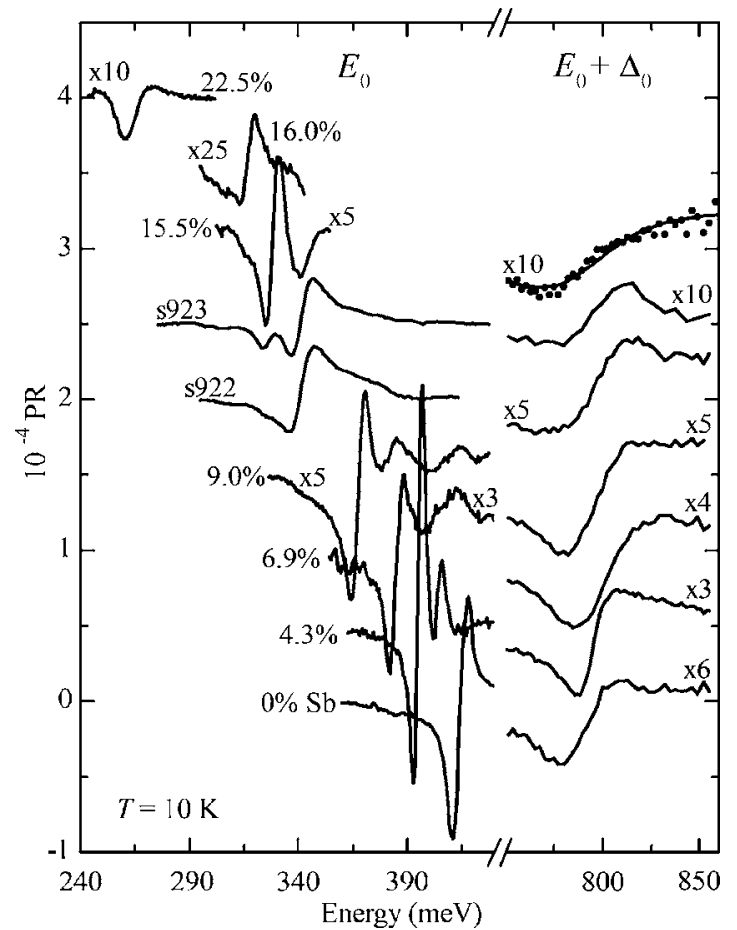

FIG. 1. Low temperature PR of the $E_{0}$ and $E_{0}+\Delta_{0}$ features in the seven InAsSb samples, as well as two pentenary GaInAsPSb samples, $s 922$ and $s 923$. For clarity, spectra are vertically offset according to Sb content shown next to each spectrum (base lines indicated by ticks on vertical axis). For the weakest observed $E_{0}+\Delta_{0}$ feature, data points with a least-squares fit are shown (see text).

only $\sim 2 \mathrm{meV}$ which we account for later. PR features from the $E_{0}$ transition in each sample are clearly visible, displaying a strong redshift with increasing antimony concentration. The corresponding transition energies were determined precisely by fitting all the PR features with third-derivative functional forms (TDFFs). ${ }^{19}$ In the case of the $E_{0}$ feature, the resulting fitted energies agree to within a few $\mathrm{meV}$ of the peak positions of the corresponding $10 \mathrm{~K}$ photoluminescence (PL) spectra (not shown). We find that the sample with the highest Sb content $(22.5 \%)$ has $E_{0}=261 \pm 5 \mathrm{meV}(4.75 \mu \mathrm{m})$, which represents the longest wavelength PR signal measured so far using grating spectroscopy. ${ }^{20,21}$ The PR signal generally gradually decreases with increasing Sb content (see multiplication factors in Fig. 1). However, the $E_{0}$ PR feature remains quite sharp, with width ranging from 7 to just $23 \mathrm{meV}$, indicating high-quality epilayers and enabling a more precise determination of $E_{0}$ than is possible from the corresponding $10 \mathrm{~K} \mathrm{PL}$ peaks (widths ranging from 20 to $32 \mathrm{meV}$ ).

For all but the highest two $\mathrm{Sb}$ concentrations we also observe a PR feature in the $800 \mathrm{meV}$ near-infrared region, which we believe is associated with the $E_{0}+\Delta_{0}$ transition. Again, the energies of these features were determined precisely by fitting with a TDFF, as shown in the example in Fig. 1 for the $15.5 \% \mathrm{Sb}$ sample. For $\mathrm{InAs}_{1-x} \mathrm{Sb}_{x}$ samples grown on InAs there is a possibility that these features may be partially, or entirely, due to the $E_{0}+\Delta_{0}$ transition of the substrate layer, rather than the epilayer. However, we are confident that this is not the case here since the penetration depth of the HeNe laser through the $500 \mathrm{~nm}$ thick InAs ${ }_{1-x} \mathrm{Sb}_{x}$ epilayers is $\sim 100 \mathrm{~nm}$ at $10 \mathrm{~K}$, making modulation of the substrate unlikely. Furthermore, since we do not observe sigDownloaded 30 Mar 2009 to 131.227.178.132. Redistribution subject

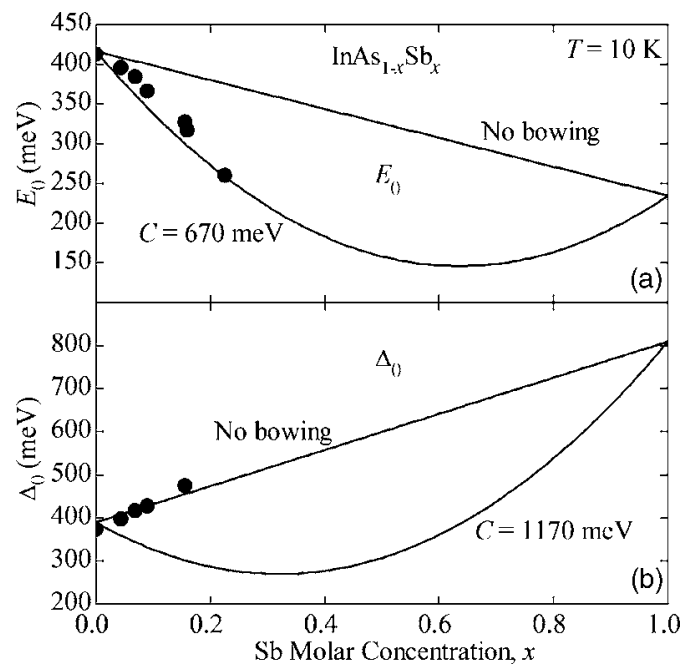

FIG. 2. Variation of the fundamental band gap $E_{0}$ (a) and spin-orbit splitting energy $\Delta_{0}$ (b) at $10 \mathrm{~K}$ as a function of $\mathrm{Sb}$ composition for $\mathrm{InAs}_{1-x} \mathrm{Sb}_{x}$ as determined from the PR spectra of Fig. 1. Estimated uncertainties due to the fitting process are smaller than the symbols used in both graphs. The lines and curves are explained in the text.

nificant PR signals at $10 \mathrm{~K}$ near the InAs band gap $\left(E_{0} \sim 415 \mathrm{meV}\right)$, we would not expect to observe a corresponding $E_{0}+\Delta_{0}$ substrate signal since the penetration depth of the probe beam in $\operatorname{InAs}_{1-x} \mathrm{Sb}_{x}$ at $800 \mathrm{meV}$ is less than half of that at $400 \mathrm{meV}$.

The PR spectra of the two InAsSb-rich $\mathrm{Ga}_{z} \mathrm{In}_{1-z} \mathrm{As}_{1-y-x} \mathrm{P}_{y} \mathrm{Sb}_{x}$ LPE samples (s922 and s923) also show features near $800 \mathrm{meV}$ in Fig. 1, similar to those of $\mathrm{InAs}_{1-x} \mathrm{Sb}_{x}$. Since these were grown on $\mathrm{GaSb}$ and not on InAs, such features cannot arise from $E_{0}+\Delta_{0}$ substrate transitions. On the other hand, at $10 \mathrm{~K} \mathrm{GaSb}$ has a fundamental band gap of $812 \mathrm{meV} .{ }^{10}$ However, this is more than $15 \mathrm{meV}$ above the fitted energies obtained for the PR of $s 922$ and $s 923$ in this spectral region. This, combined with the fact that the pentenary epilayers are $\sim 1.5 \mu \mathrm{m}$ thick in these LPE samples (over ten times the penetration depth of the $\mathrm{HeNe}$ laser), rules out the possibility that the observed features near $800 \mathrm{meV}$ arise from $E_{0}$ substrate signals.

Figure 2(a) shows the $10 \mathrm{~K}$ values for $E_{0}$ obtained from the TDFF fits of the $\operatorname{InAs}_{1-x} \mathrm{Sb}_{x}$ PR spectra in Fig. 1, as a function of $\mathrm{Sb}$ content. The straight line shows a linear interpolation (no bowing) between the InAs and InSb end points, while the curve represents the accepted bowing of $C=+670 \mathrm{meV} .{ }^{10}$ Our values for $E_{0}$ tend to lie slightly above the accepted bowing curve, by $\sim 20 \mathrm{meV}$, similar to Ref. 9 and other studies on InAsSb samples rich in InAs. ${ }^{7,8}$ This may be due to a slight overestimation of the Sb concentrations due to our assumption of full relaxation.

Figure 2(b) shows our $10 \mathrm{~K} \Delta_{0}$ results for the $\operatorname{InAs}_{1-x} \mathrm{Sb}_{x}$ samples where an $E_{0}+\Delta_{0}$ PR feature was observed. It is clear that $\Delta_{0}$ does not exhibit the strong, positive bowing claimed by Berolo and Woolley ${ }^{11}$ and quoted by Vurgaftmann et al. [ $C=+1170 \mathrm{meV}$, the curve in Fig. 2(b)]. Our results show no positive bowing, and, if anything, may show a slightly opposing trend with a fit of the data in Fig. 2(b) (not shown) giving $C\left(\Delta_{0}\right)=-225 \mathrm{meV}$. Although a negative bowing has been predicted by Wei and Zunger for ordered ternary alloys, ${ }^{18}$ our result provides only tentative supporting evidence of this due to the range of Sb content studied. However, our work on high-quality, epitaxial MBE $\operatorname{InAs}_{1-x} \mathrm{Sb}_{x}$ to AIP license or copyright; see http://apl.aip.org/apl/copyright.jsp 
samples supports the contention of Ref. 18 that the strong, positive $\Delta_{0}$ bowing of Ref. 11 may indeed be due to the inhomogeneity in their Bridgman-grown samples.

A similar TDFF fitting analysis of the PR spectra of the two $\mathrm{Ga}_{z} \mathrm{In}_{1-z} \mathrm{As}_{1-y-x} \mathrm{P}_{y} \mathrm{Sb}_{x}$ LPE samples in Fig. 1 ( $s 922$ and $s 923)$ gives $\Delta_{0}$ values of $453 \pm 5$ and $460 \pm 5 \mathrm{meV}$, respectively. We can test whether these values are also consistent with a negligible $\Delta_{0}$ bowing in $\operatorname{InAs}_{1-x} \mathrm{Sb}_{x}$ by interpolating to find the expected pentenary values, $P$. We do this by treating these samples as a mixture of two quaternary alloys: $Q_{1}=\mathrm{GaAs}_{1-y-x} \mathrm{P}_{y} \mathrm{Sb}_{x}$ and $Q_{2}=\operatorname{InAs}_{1-y-x} \mathrm{P}_{x} \mathrm{Sb}_{x}$. We use the interpolation formula described by Krijn ${ }^{22}$ to calculate these and then interpolate between them using $P=z Q_{1}+(1-z) Q_{2}$ $-z(1-z) C^{\prime}$, where $z$ is the gallium content and $C^{\prime}=(1-y$ $-x) C_{\mathrm{InGaAs}}+y C_{\mathrm{InGaP}}+x C_{\mathrm{InGaSb}}$. Using parameters from Ref. 10 and including the value $C_{\mathrm{InGaSb}}=+1170 \mathrm{meV}$ of Berolo and Woolley ${ }^{11}$ would give $\Delta_{0}=293 \pm 20$ and $286 \pm 20 \mathrm{meV}$ for $s 922$ and $s 923$, respectively, where the errors arise from the compositional uncertainties of the pentenary alloys. These predicted values are very much lower than our measured values. However, a zero bowing, $C_{\mathrm{InGaSb}}=0 \mathrm{meV}$, yields 415 and $420 \mathrm{meV}$, respectively, much closer to our measurements. This is further evidence that $\Delta_{0}$ exhibits negligible positive bowing in $\operatorname{InAs}_{1-x} \mathrm{Sb}_{x}$. Note that our tentative fitted bowing of $C_{\mathrm{InGaSb}}=-225 \mathrm{meV}$ predicts $\Delta_{0}(s 922)$ $=438 \mathrm{meV}$ and $\Delta_{0}(s 923)=446 \mathrm{meV}$, equal to the corresponding measurements within the aforementioned uncertainties.

To put our results into context, we can consider their impact on predicting Auger recombination rates in, say, a cryogenically cooled InAs ${ }_{1-x} \mathrm{Sb}_{x}$ based device for $\mathrm{CO}_{2}$ gas detection at $4.25 \mu \mathrm{m}\left(E_{0} \sim 290 \mathrm{meV}\right)$, which from Fig. 2(a) would be provided by an $\mathrm{Sb}$ molar fraction of $\sim 17 \%$. Currently accepted literature results would predict $\Delta_{0} \sim 295 \mathrm{meV},{ }^{10,11}$ within $5 \mathrm{meV}$ of $E_{0}$. Such a resonance, $E_{0} \approx \Delta_{0}$ would result in very high CHSH Auger losses, since near-vertical transitions could occur between states close to the band edges. ${ }^{3}$ However, using our result of negligible bowing gives $\Delta_{0} \sim 460 \mathrm{meV}$, which, since $\Delta_{0} \gg E_{0}$ would predict that the CHSH process is no longer of importance compared to the other processes. ${ }^{3}$ Therefore, theoretical predictions of the performance of such a device would differ greatly in terms of the extent of the different Auger losses, depending on which value for $\Delta_{0}$ was used.

In the future we hope to extend these PR studies to even longer wavelengths, in order to quantify $C\left(\Delta_{0}\right)$ in $\operatorname{InAs}_{1-x} \mathrm{Sb}_{x}$ samples containing higher antimony fractions.

The authors thank EPSRC (GR/S08817/01) for support and studentships for two of the authors (S.A.C. and P.J.B.), the Royal Society UK-Taiwan International Joint Academic Exchange Project NC/TW JP03-05/15628, and the National Science Council of the Republic of China (NSC-94-2215-E002-016).

${ }^{1}$ A. Joullie and P. Christol, C. R. Phys. 4, 621 (2003).

${ }^{2}$ S. R. Kurtz, R. M. Biefeld, A. A. Allerman, A. J. Howard, M. H. Crawford, and M. W. Pelczynski, Appl. Phys. Lett. 68, 1332 (1996).

${ }^{3}$ A. Haug, J. Phys. C 16, 4159 (1983).

${ }^{4}$ A. Rakovska, V. Berger, X. Marcadet, B. Vinter, G. Glastre, T. Oksenhendler, and D. Kaplan, Appl. Phys. Lett. 77, 397 (2000).

${ }^{5}$ A. Krier, M. Stone, Q. D. Zhuang, Po-Wei Liu, G. Tsai, and H. H. Lin, Appl. Phys. Lett. 89, 091110 (2006).

${ }^{6}$ J. A. Van Vechten and T. K. Bergstresser, Phys. Rev. B 1, 3351 (1970).

${ }^{7}$ A. G. Thompson and J. C. Woolley, Can. J. Phys. 45, 2597 (1967).

${ }^{8}$ M. Y. Yen, R. People, and K. W. Wecht, J. Appl. Phys. 64, 952 (1988).

${ }^{9}$ Z. M. Fang, K. Y. Ma, D. H. Jaw, R. M. Cohen, and G. B. Stringfellow, J. Appl. Phys. 67, 7034 (1990).

${ }^{10}$ I. Vurgaftman, J. R. Meyer, and L. R. Ram-Mohan, J. Appl. Phys. 89, 5815 (2001).

${ }^{11}$ O. Berolo and J. C. Woolley, Proceeding of the 11th International Conference on the Physics of Semiconductors (ICPS), Warsaw, 1972, p. 1420 .

${ }^{12}$ W. M. Coderre and J. C. Woolley, Can. J. Phys. 46, 1207 (1968).

${ }^{13}$ J. A. Van Vechten, O. Berolo, and J. C. Woolley, Phys. Rev. Lett. 29, 1400 (1972).

${ }^{14}$ E. D. Siggia, Phys. Rev. B 10, 5147 (1974).

${ }^{15}$ P. Parayanthal, C. S. Ro, F. H. Pollak, C. R. Stanley, G. W. Wicks, andj L. F. Eastman, Appl. Phys. Lett. 43, 109 (1983).

${ }^{16}$ E. H. Perea, E. E. Mendez, and C. G. Fonstad, Appl. Phys. Lett. 36, 978 (1980).

${ }^{17}$ P. Parayanthal and F. H. Pollak, Phys. Rev. B 28, 3632 (1983).

${ }^{18}$ S. Wei and A. Zunger, Phys. Rev. B 39, 6279 (1989).

${ }^{19}$ D. E. Aspnes, Surf. Sci. 37, 418 (1973).

${ }^{20}$ C. H. Lin, K. E. Singer, J. H. Evans-Freeman, K. Heath, and M. Missous, Semicond. Sci. Technol. 12, 1619 (1997).

${ }^{21}$ T. J. C. Hosea, M. Merrick, and B. N. Murdin, Phys. Status Solidi A 202, 1233 (2005)

${ }^{22}$ M. P. C. M. Krijn, Semicond. Sci. Technol. 6, 27 (1991). 